\title{
Coronal restructuring and electron acceleration following a filament eruption
}

\author{
Ch. Marqué, P. Lantos, K.-L. Klein, and J. M. Delouis \\ DASOP-LPSH, Observatoire de Meudon, 92195 Meudon Cedex, France
}

Received 12 April 2001 / Accepted 9 May 2001

\begin{abstract}
We present a multi wavelength observation of a filament eruption occurring on September 2nd 1999. The slow pre-event evolution of the filament ends with energy release signaled by a faint radio burst observed with the Nançay Radioheliograph and a faint LDE. The restructuring of a large area around the site of the eruption was monitored both with SOHO/EIT observations as well as with the radio data. A moving noise storm occurred in the vicinity of the initial radio burst in coincidence with a faint coronal brightening observed with the LASCO coronagraph; no Coronal Mass Ejection was seen. On a smaller energetic scale, this event presents several manifestations of coronal disturbances observed in many CME associated events. Finally, it makes the link between previous studies on noise storm onsets and coronal restructuring.
\end{abstract}

Key words. Sun: corona - Sun: filament - Sun: radio radiation - Sun: activity

\section{Introduction}

Filament disappearances involve a large range of structures and phenomena observed at different wavelengths. They have been observed in $\mathrm{H} \alpha$ since the beginning of daily surveys of solar activity, and they have been called "Disparition brusque" (DB, d'Azambuja \& d'Azambuja 1948). In fact, this term is associated with two different classes of events: thermal events, due to simple heating of the cold and dense material of the filament, and dynamic ones (filament eruptions) involving ascending motion of material into the corona. This article deals with this second category.

Since Skylab, it has been known that prominence eruptions can be associated with magnetic reconfigurations leading to loop heating observed in X rays as LDE (Long duration Events, Pallavicini et al. 1977) and in the centimeter range as GRF (Gradual Rise and Fall, Hudson \& Ohki 1972). In addition, large scale morphological changes are generally observed in the topology of the loop systems (Uchida 1998). Coronal and interplanetary disturbances such as CMEs (Coronal Mass Ejections) are also associated with filament eruptions and they are seen both for active region filaments and quiescent filaments (Hundhausen 1999; Webb 1998).

Send offprint requests to: Ch. Marqué, e-mail: christophe.marque@obspm.fr
In the absence of $\mathrm{H} \alpha$ flare, radio observations of prominence eruptions mostly involve noise storm emissions and only a few are available. Noise storms are the most frequent non-thermal emissions in the meter and decameter range. Spectral observations show continuum emission and superimposed narrow-band and short-duration type I bursts. Noise storms are emitted by accelerated electrons in closed magnetic loops. The emission takes place at the plasma level, as attested by their high degree of polarization corresponding to O-mode (see monograph by Elgarøy 1977). Thus, the observation of noise storms at one frequency indicates the specific critical electron density.

While filaments themselves have been observed for years at centimeter and short decimeter wavelengths as depressions in brightness temperature (Hiei et al. 1986), observations performed in the meter range do not show a systematic association between filaments and radio features, source or depression (Alissandrakis \& Lantos 1996). In the long decimeter range, Delouis (1999) and Marqué et al. (1999) have recently shown for the first time, filaments as brightness depression on the disk at 410 and $327 \mathrm{MHz}$.

This paper outlines an example of filament eruption observed in $\mathrm{H} \alpha$, EUV, SXR and radio wavelengths, occurring on September 2nd 1999. After a slow evolution, a faint radio burst marks the beginning of the optical, UV and radio event. Subsequent coronal modifications are observed, such as dimmings in EUV and for the first time, in radio, as well as a noise storm onset. The paper is organized as 


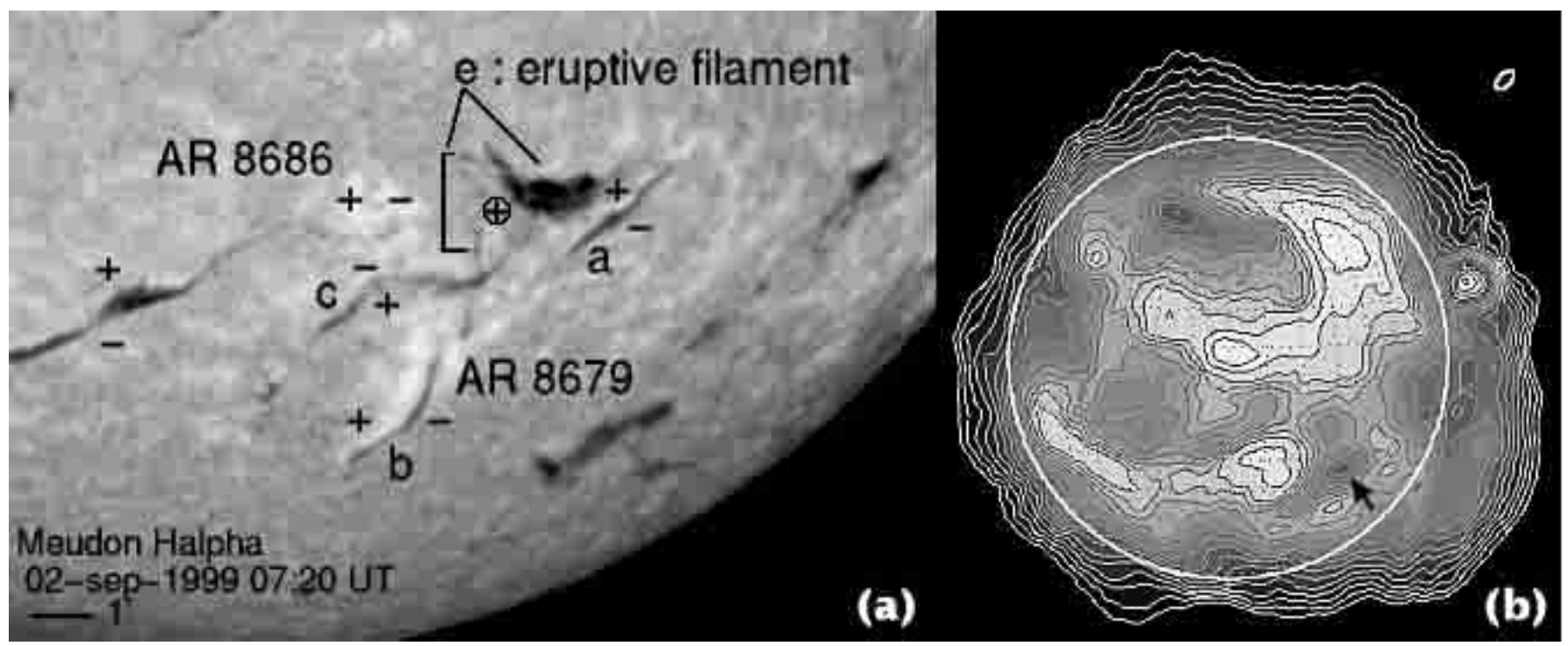

Fig. 1. a) Meudon $\mathrm{H} \alpha$ overview of the pre-eruptive region at 07:20 UT. Active region numbers are given by NOAA. The magnetic field polarity is marked as indicated by SOHO/MDI. The circled cross indicates the mean polarity of the underlying area without a strong magnetic gradient. b) Synthesis map performed between 08:22 UT and 10:22 UT at $327 \mathrm{MHz}(92 \mathrm{~cm}-\lambda)$. The depression associated with the filament is pointed out by the arrow on the decimetric map. The beam of the telescope is indicated to the upper right.

follows: we present a brief overview of the instruments and radio imaging methods (Sect. 2), then give an extensive multi wavelength description of the event (Sect. 3), we propose an interpretation in terms of large scale coronal modifications (Sect. 4), and finally give some concluding remarks.

\section{Instruments}

The Nançay Radioheliograph (hereafter NRH) is a T-shape interferometer operating in the meter and long decimeter range (150-450 MHz). It has been upgraded to provide high-time resolution 2d-images (Kerdraon \& Delouis 1997). In November 1998, an antenna was added to the North-South branch, giving a twofold better resolving power. To monitor the sporadic activity of the sun, such as radio bursts, intensity and polarization images are produced at a rate up to $8 \mathrm{im} / \mathrm{s}$. Table 1 summarizes the technical characteristics of the NRH. The resolution is given for each of the 1-d arrays; instantaneous 2-d images have a lower resolution, because the longer baselines are not currently used.

Using an aperture synthesis mode, we can also study quiet sun features such as coronal holes, streamers or the coronal plateau, which is the radio counterpart of the streamer belt that surrounds the Sun (Lantos \& Alissandrakis 1999). The Earth rotation provides a better Fourier plane coverage and thus the quality of radio maps is improved as well as the sensitivity. Nevertheless, moderate or strong permanent noise storms can prevent the calculation of reliable synthesis maps since they have high temporal variations in flux and brightness temperature. We use the two modes of data reduction in this article, producing a pre-event 2 hour synthesis radio map
Table 1. Technical overview of the NRH.

Stand. freq. (MHz): 164.0, 236.6, 327.0, 410.5, 432

Stand. wavelengths (m): 1.83, 1.27, 0.92, 0.73, 0.69

E-W array: 16 "wide band" dipoles $+2 \times 10 \mathrm{~m}$ dishes $+1 \times 7 \mathrm{~m}$ dish

Baseline: $50 \mathrm{~m}$ to $3200 \mathrm{~m}$

Resolution: 1.4' (164 MHz) to $31^{\prime \prime}$ (432 MHz)

N-S array: $24 \times 5 \mathrm{~m}$ dishes $+1 \times 7 \mathrm{~m}$ dish (since Nov. 98)

Baseline: $54 \mathrm{~m}$ to $2400 \mathrm{~m}$

Resolution: $3.4^{\prime}$ (164 MHz) to $1.3^{\prime}(432 \mathrm{MHz})\left(\delta=23^{\circ}\right)$

in order to show the main solar features, and we follow the eruption with high time resolution images (essentially 2 images/s and 1 image/128 s). On the chosen day, the pointing accuracy was limited to $0.05 R_{\odot}$, about $50^{\prime \prime}$.

In addition to radio data, detailed coronal images are available for this event. Large-scale coronal images were provided by the LASCO-C2 instrument (Brueckner et al. 1995) on board the SOHO spacecraft. SOHO/EIT (Delaboudinière et al. 1995) was used in order to follow the dynamical behavior of the region under study in EUV, as well as YОНКОН/SXT (Acton et al. 1988) in soft X-rays. Photospheric magnetic field observations are available from Kitt Peak and Mt Wilson (Solar Geophysical Data, 1999) as well as from the SOHO/MDI experiment (Scherrer et al. 1995). The Meudon Observatory provided Ca II K1v and $\mathrm{H} \alpha$ spectroheliograms, as well as an $\mathrm{H} \alpha$ movie (wings and center of line). 


\section{Observations}

On September 2nd, 1999, after 11:45 UT, a filament erupted in the south-western quadrant of the disk. The eruption was preceded by various manifestations of EUV activity in a wide region around the filament, and followed by a faint ejecta and energy release in the vicinity of the filament, including the acceleration of electrons which produced decimetric and metric radio emissions and, judging from the time history of whole-Sun fluxes, plasma heating. We first describe the context where the eruption occurred, then discuss the dynamical disappearance of the filament and changes in its environment and the overlying corona, and finally present the observations of electron acceleration and plasma heating and their timing with respect to the eruptive event.

\subsection{Overview: Filaments and active regions prior to eruption}

Figure 1a shows the filament which eventually erupted (labelled e) and its environment, about four hours before the eruption. The filament is broad and dark with recognizable internal structure. Unlike other filaments, it does not overlie a well-defined line of photospheric magnetic field reversal, but a patchwork of weak magnetic polarities. Three other filaments (a-c) lie in its surroundings, as well as two active regions (NOAA 8686: South 24 West 19; NOAA 8679: South 34 West 14).

Filament e appears near 13 UT on August 31st, immediately north of filaments a and c. It has two components extending over $\sim 13^{\circ}$ in longitude on September 1st. AR 8686, east of filament e, grew in size and complexity since its birth on August 28th, as shown by YOHKOH/SXT observations and by the appearance of a new sunspot on September 1st. Between September 1st and 2nd filament e became more compact in the east-west direction, and broader in the north-south direction. Its trailing part was bent northward as seen in Fig. 1. This is probably evidence that new flux emerging in AR 8686 pushed magnetic structures ahead of the active region further to the west.

Before its eruption, filament e was observed in absorption in coronal lines of the EIT instrument, Fe XII, Fe XV, Fe IX/X. The eruptive phenomenon was monitored in the Fe XII line every 15 min.

Radio observations performed with the NRH before the eruption show a clear depression in the vicinity of filament $\mathbf{e}$, in the decimetric range $(410 \mathrm{MHz}$ and $327 \mathrm{MHz}$, respectively $73 \mathrm{~cm}-\lambda$ and $92 \mathrm{~cm}-\lambda)$. The radio dip is an elongated structure overlaying the area between filament e and the west part of filament $\mathbf{c}$. The minimum of the depression, indicated by an arrow in Fig. 1b is centered over filament e. No clear dip is detected on metric maps (not shown here) at 236.6 and $164 \mathrm{MHz}(127 \mathrm{~cm}-\lambda$ and $183 \mathrm{~cm}-\lambda)$; while filament $\mathbf{e}$ is invisible at $164 \mathrm{MHz}$, a faint decrease in brightness temperature is observed at 236.6 MHz in its vicinity. Other features on the decimetric

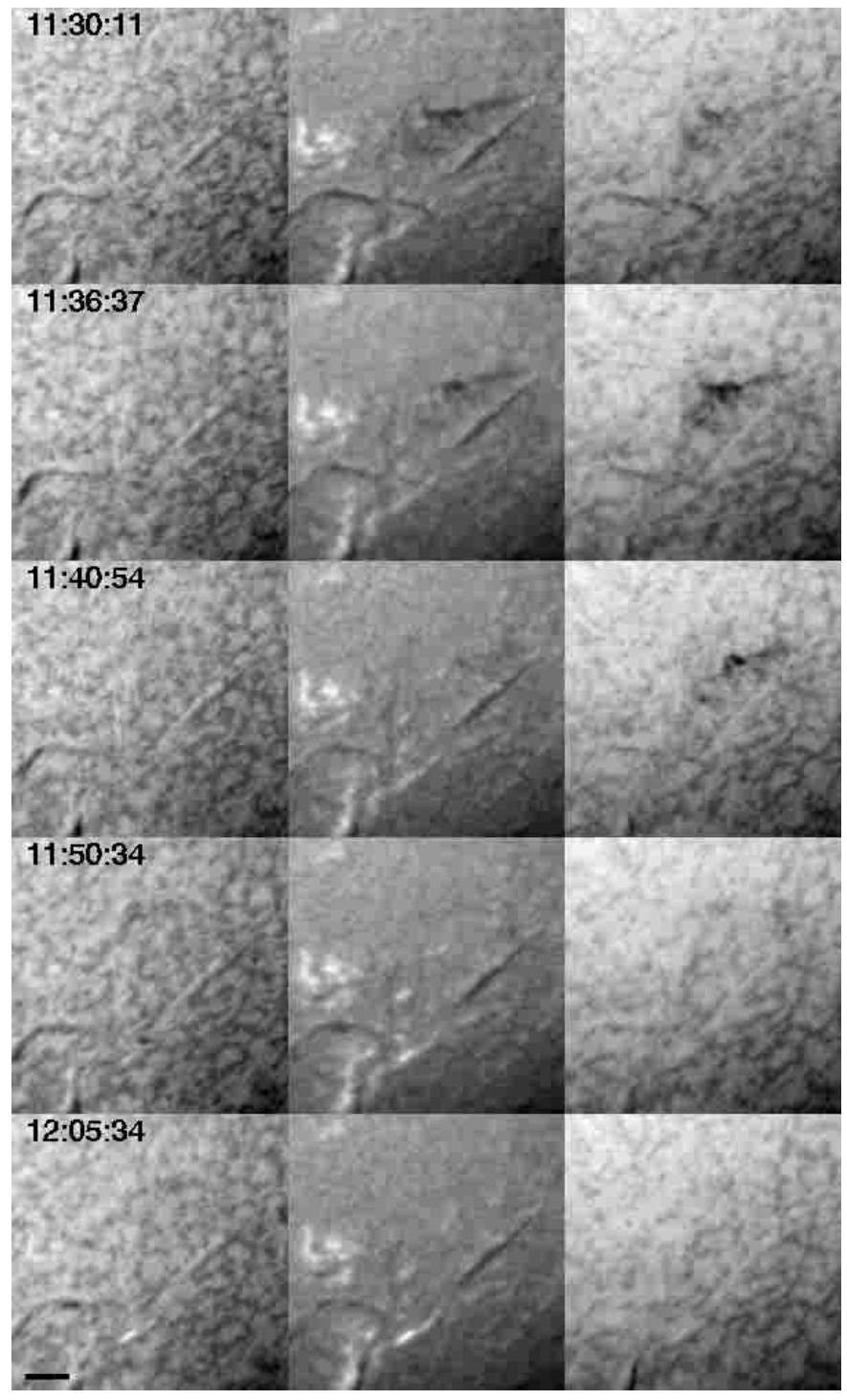

Fig. 2. Eruption of filament e in three positions in the $\mathrm{H} \alpha$ line. Left-hand column: $\mathrm{H} \alpha+0.5 \AA$, middle: $\mathrm{H} \alpha$, right-hand: $\mathrm{H} \alpha-0.5 \AA$. The thick horizontal line is a length-scale of $1^{\prime}$.

map presented here are coronal holes (polar and equatorial holes on east limb), thermal emission from unresolved loops in the vicinity of AR 8686, and a faint non-thermal radio source above the west limb, probably a decaying noise storm born on August 29th on the disk.

\subsection{The eruptive event}

Filament e, as observed in the $\mathrm{H} \alpha$ line, erupted between 11:40 and 11:47. We will hereafter consider a reference time $T_{0}=11: 45$ UT for sorting the different steps of the event.

\subsubsection{The dynamical phase}

The dynamical disappearance of filament $\mathbf{e}$ is illustrated in the series of images in the center and wings of the $\mathrm{H} \alpha$ 
line in Fig. 2. The $\mathrm{H} \alpha$ movie shows that the filament expands and possibly rotates around its main axis between 9:15 and 10:00 UT. Parts of the structure became visible in the blue wing during this period. After $10 \mathrm{UT}$ the filament consisted of two parallel roughly east-west directed lanes. The southern one disappeared without becoming visible in the wings. The northern part gradually appeared in the blue wing since about 11:30 UT, but not in the red wing. This is an additional indication that the filament was rising. It disappeared from line center between 11:40 and 11:47 UT. Signatures in the blue wing are observed until $\sim 12: 30$, but the orientation changed from predominantly east-west to north-south (e.g., 11:50 UT in Fig. 2), and the feature moves north-westward in projection on the disk. This shows that filament e undergoes a dynamical disappearance and is pushed towards north-west. The $\mathrm{H} \alpha$ movie shows an initially slow north-westward motion which accelerated between 11:40 and 11:45 UT, just before the filament disappeared from the line center image. EIT images show first the eruption of the filament towards the north-west, and eventually its fall and destruction, back to the surface.

The dynamical DB of filament e affects filament $\mathbf{c}$ where conspicuous, apparently horizontal, motions are observed beginning at 10:00 UT. After 11:00 UT ( $\left.T_{0}-45 \mathrm{mn}\right)$ EIT observes bright elongated structures overlying the $\mathrm{H} \alpha$ filament. These bright structures either reveal the heated upper part of the filament $\left(T \sim 1.5 \times 10^{6} \mathrm{~K}\right)$, i.e. a partial thermal disappearance of the filament, analogous to the case reported by Khan et al. (1998). Alternatively, the bright structures can be sheared loops above the filament, activated before the eruption of filament e. Filament $\mathbf{c}$ eventually partially disappears during the eruption of filament e. Its disappearance is probably thermal, since observations in the blue wing of $\mathrm{H} \alpha$ reveal no significant motions. The EIT movie shows the time correlation of the thermal disappearance of filament $\mathbf{c}$ and the dynamical one of filament e. Despite its proximity to filament $\mathbf{e}$, filament $\mathbf{a}$ is not affected by the eruption and does not present any disturbances or activity triggered by the event.

A wide range of surrounding magnetic structures was affected in the course of the eruption: a faint $\mathrm{H} \alpha$ ribbon appeared at the western side of filament $\mathbf{c}$ at 12:00 $\left(T_{0}+15 \mathrm{mn}\right)$, see Fig. 2. After $13 \mathrm{UT}$, EIT saw arcades brightening in both active regions 8686 and 8679, and loops (apparently non potential) interconnecting the two active regions.

Much wider magnetic structures are involved in this event. Prior to the eruption, large scale dynamical perturbations are observed in the Fe XII line of the EIT instrument at 11:00 UT $\left(T_{0}-45 \mathrm{mn}\right)$, when transequatorial loops linking AR 8686 and AR 8677 in the northern hemisphere begin to kink. As illustrated in Fig. 3, a propagating feature starts from the south (AR 8686) at this time and reaches the north $(\mathrm{AR} 8677)$ at 11:48 $\mathrm{UT}\left(T_{0}+3 \mathrm{mn}\right)$.

Observations performed with EIT at $284 \AA$ (FeXV), despite a poor time resolution, show significant differences between the pre event and post event configuration. In

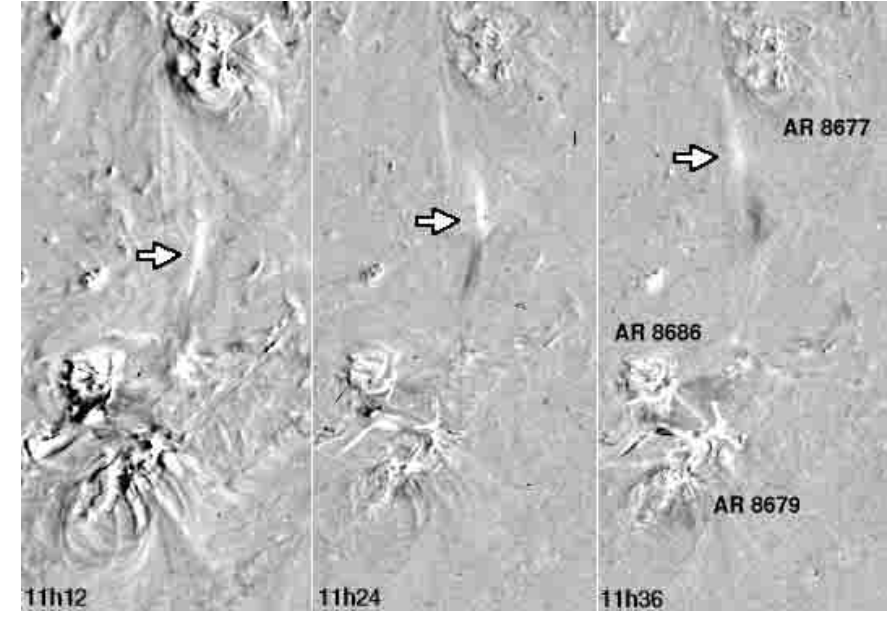

Fig. 3. SOHO/EIT running difference images of a pre-eruptive event affecting a transequatorial loop between AR 8686 (south) and 8677 (north); three different times are presented 11:12, 11:24 and 11:36 UT (respect. $T_{0}-33, T_{0}-21$ and $T_{0}-09 \mathrm{mn}$ ). The moving structure is indicated by the arrow. The same loop system is affected by the eruption of filament $\mathbf{e}$.

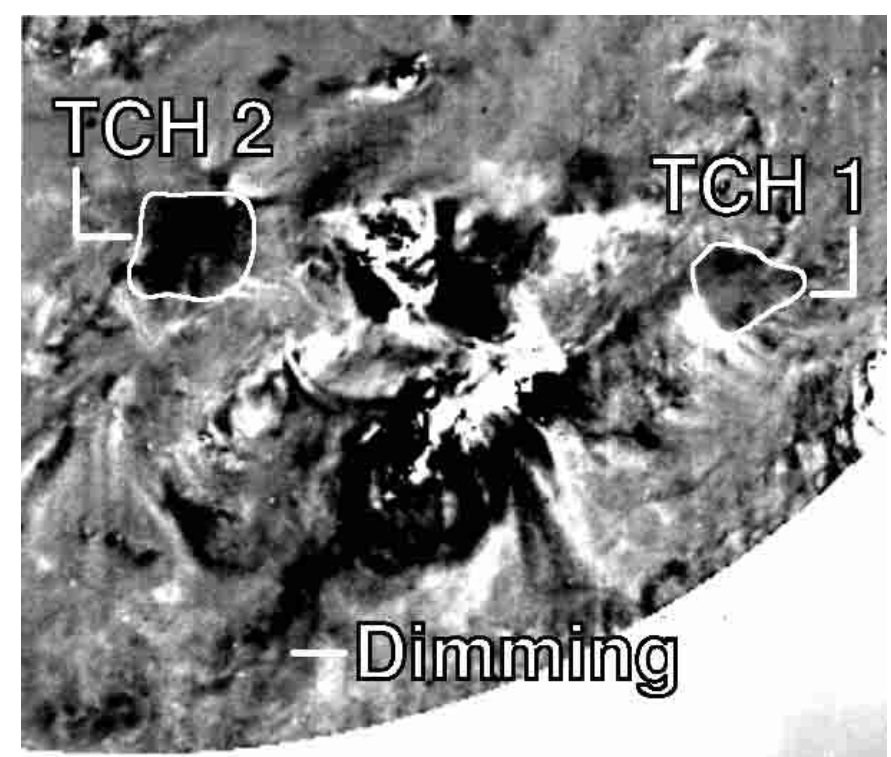

Fig. 4. Difference of two EIT images in the Fe XII line at 10:00 and 14:00 UT. The former image has been corrected for the differential solar rotation. Transient Coronal Holes (TCH1 and $\mathrm{TCH} 2$ ) are indicated as well as the area of the dimming mentioned in the article.

this line (also at $195 \AA$ ) Transient Coronal Holes appear far away from the two AR, as new dimmings, but they were already visible on YОНКОН observations performed before the event. Figure 4 shows the location of the two transient corona holes, with difference images techniques applied to EIT images at $195 \AA$ (Fe XII), and taking into account the solar rotation.

Radio manifestations of the event imply non thermal emissions which will be discussed in the next section, and 


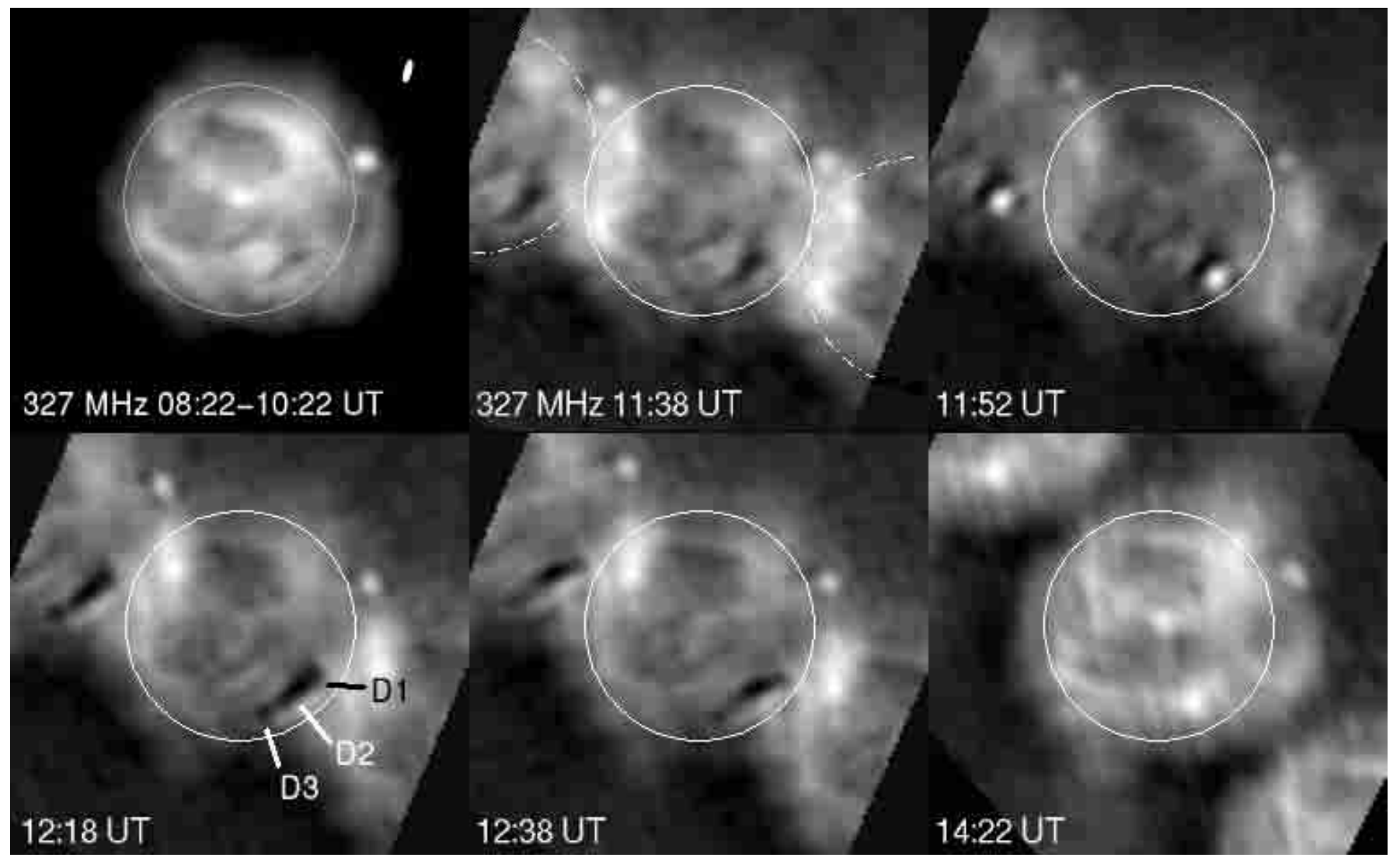

Fig. 5. Upper left image is a pre-eruptive synthesis radio map at $327 \mathrm{MHz}(\sim 92 \mathrm{~cm}-\lambda)$ between 08:22 and 10:22 UT. The optical limb is indicated by the white circle. The beam of the telescope is in the upper right corner. Other images show the evolution of the event with time. The difference size and depth of the depression between the synthesis map and the next image (upper middle) is partially due to image calculation. The burst A (see text) appears on upper right image. Aliasing, as indicated by dash-dot circles, due to the array configuration, affects these raw images and explains why different images of the sun partly overlap. The synthesis map (upper left image) does not suffer from such artifacts but cannot monitor the event with time. D1, D2, D3 point out the three substructures within the main radio dimming (see text and Fig. 6 for more details).

an expanding radio dimming from the location of the initial depression. Figure 5 illustrates different steps of the eruption at $327 \mathrm{MHz}$ with instantaneous 2-d images, compared to a pre-event synthesis map computed over two hours (upper left image). The first two upper maps are pre eruptive images; the main differences between them arise from computational techniques, especially the visibility of the depression which depends on the beam orientation, and the accuracy of the quiet sun component measurement. As the optical filament erupts, a radio burst occurs (upper right image), and the width, the length and the depth of the initial radio depression increase. Figure 6 presents the temporal evolution of the length and depth of the radio depression at $410.5 \mathrm{MHz}$. For each available image, a profile of minimum brightness was calculated as a function of distance, along the main axis of the depression. Successive profiles taken during the whole observing period were combined to give the grey scale plot in Fig. 6. Three substructures are observed within the main dip, and are denoted D1, D2 and D3. The total length of the dip is roughly $0.7 R_{\odot}$, compared to the size of the pre-eruption depression $\sim 0.2 R_{\odot}$. The north western part of the radio depression (D1) expands from the time of the burst up to 13:00 UT. The main part of the radio depression is rather static: the D2 dip is located along the optical filament $\mathbf{a}$ (with a pointing accuracy of $\sim 0.05 R_{\odot}$ ). The last dip (D3) appears in the vicinity of a dimming area seen with EIT during the eruption (see Fig. 4) and disappears after 13:00 UT. The radio depression $(\mathrm{D} 1+\mathrm{D} 2+\mathrm{D} 3)$ slowly vanishes after 13:30 UT and is not seen after 14:00 $\left(\sim T_{0}+2 \mathrm{~h}\right)$.

\subsubsection{Energy release}

The localized source in the radio image at 11:52 UT in Fig. 5 is part of a burst of $5-10$ min duration observed at all NRH frequencies and called hereafter burst A. It started at $T_{0}+3 \mathrm{mn}$, i.e. during the rapid disappearance of the $\mathrm{H} \alpha$ filament. Peak brightness temperatures are $8 \times 10^{6} \mathrm{~K}$ $(164 \mathrm{MHz}), 7 \times 10^{6} \mathrm{~K}(236.6 \mathrm{MHz}), 5 \times 10^{6} \mathrm{~K}(327 \mathrm{MHz})$, and $<5 \times 10^{6} \mathrm{~K}(410.5 \mathrm{MHz})$. They are too weak to make the burst observable by spectrographs recording the whole Sun flux. The time history of the radio emission, plotted in the upper panel of Fig. 7, suggests that burst A is a broad 


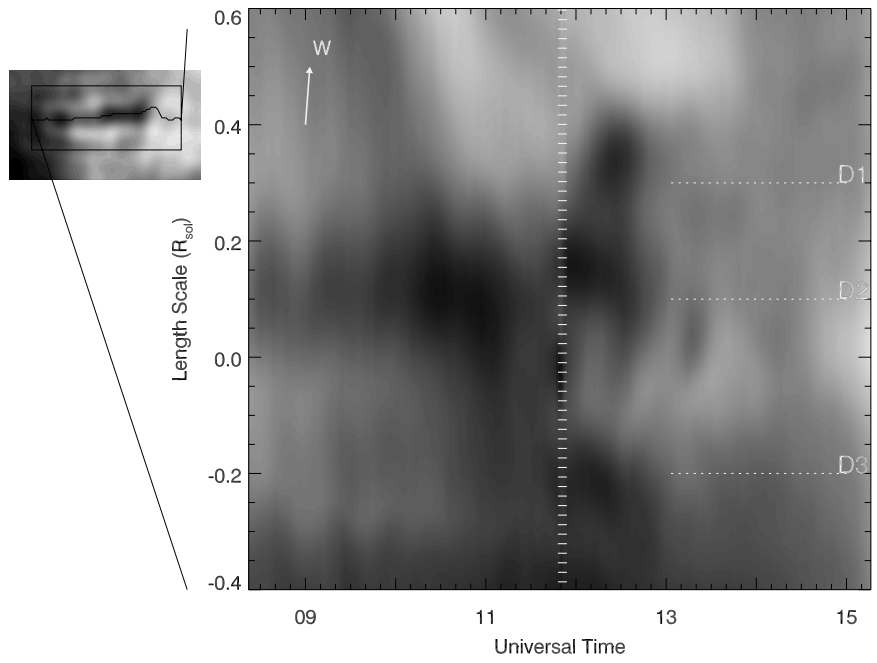

Fig. 6. Evolution with time of the length and depth of the radio depression along its main axis. On the right side are indicated the three substructures born after the eruption. The hatched area, about 11:50 UT, delineates the burst phase while the arrow points towards the West direction. On the left, the stamp image illustrates the method. The box delineates the area where the analysis was performed. A profile, along the main axis of the depression, is drawn (see text for more details).

band continuum. At $164 \mathrm{MHz}$, the burst is followed by a prolonged emission including rapid fluctuations. The time profile is typical of noise storms, revealing the acceleration of electrons to supra thermal energies which often occurs near quiescent active regions.

The whole radio event is accompanied by a soft $\mathrm{X}$-ray long duration event. The soft X-ray burst observed between 12:30 and 12:40 UT does not seem to refer to the present event (lower panel of Fig. 7). The radio burst occurs during the rise of the soft X-ray flux, while the noise storm accompanies the decay. No $\mathrm{H} \alpha$ flare is reported between 11:30 and 14 UT (SGD 667-II). Soft $\mathrm{X}$-ray structures in images obtained with $\mathrm{YOHKOH} / \mathrm{SXT}$ may be related to this LDE. New loops are seen linking both AR 8686 and AR 8679 as well as the surroundings of filament a. A global brightening of AR 8679 and a southward area is also observed during the LDE. The decreasing phase of the LDE ends after 13:20 UT, approximately 10 min before the end of the noise storm. We will discuss the possible relationships later.

The noise storm at $164 \mathrm{MHz}$ starts at the same location as the metric component of burst $A$. The source drifts outward through the corona for about $30 \mathrm{mn}$ and then remains at an approximately stable position for one more hour, where the emission eventually fades. The drift speed of the source inferred from a linear fit to the height-time plot of the radio source centroid in the bottom panel of Fig. 8 is $91 \pm 8 \mathrm{kms}^{-1}$.

Radially above the noise storm, a faint rising feature appears above the occulting disk of the LASCO-C2
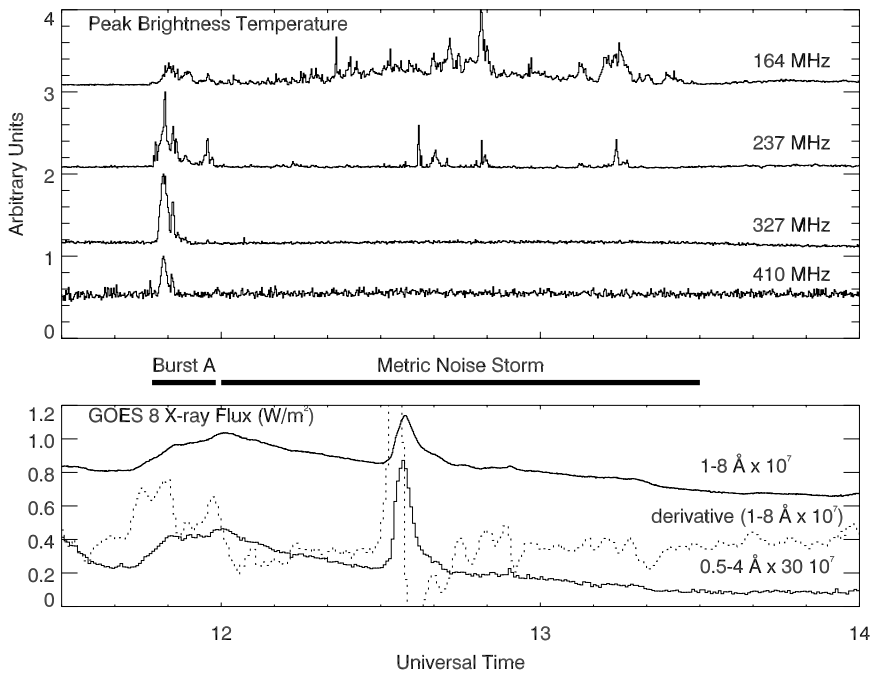

Fig. 7. Time profile of the peak brightness temperature at four frequencies, showing the initial broad band burst phase (burst A) and the noise storm at $182 \mathrm{~cm}-\lambda$. The lower panel presents the X-ray flux curve as observed by GOES 8 within two channels: $1-8 \AA$ and $0.5-4 \AA$. The dashed line is the derivative of the upper X-ray curve, giving the time of the energy release. The peak in GOES curves between 12:30 and 12:40 is not associated with our event.

coronagraph at 12:30 UT, in a region where no white light structure was seen before. The noise storm source is superposed on a LASCO difference image in the top panel of Fig. 8. The coronagraphic feature is very faint, but its centroid can be tracked in four difference images. The projected heliocentric distance plotted in the bottom panel of Fig. 8 increases at virtually the same speed $\left(92 \pm 18 \mathrm{~km} \mathrm{~s}^{-1}\right)$ as the underlying noise storm, but over a longer duration. As no depression is observed on difference images at the previous location of the LASCO feature, it is not an outgoing blob of plasma. On raw images, the LASCO feature is rather the front of a broad swelling structure, invading the higher corona, but it does not look like an usual jet or streamer. The position angle and direction of motion of the noise storm and the white-light feature are clearly different from the $\mathrm{H} \alpha$ filament, but seem to correspond to the loops south of AR 8679 (Fig. 9).

\section{Interpretation}

The eruption of filament e reveals large scale magnetic reconfigurations, implying reconnection processes as seen by the non thermal radio emission, and opening of magnetic field lines as revealed by the dimmings.

\subsection{Radio burst $A$ and the eruption}

EIT movies of the event suggest the implication of large scale arcades overlying both filament a and possibly filament e. Radio positions of the burst (see Fig. 9) with 

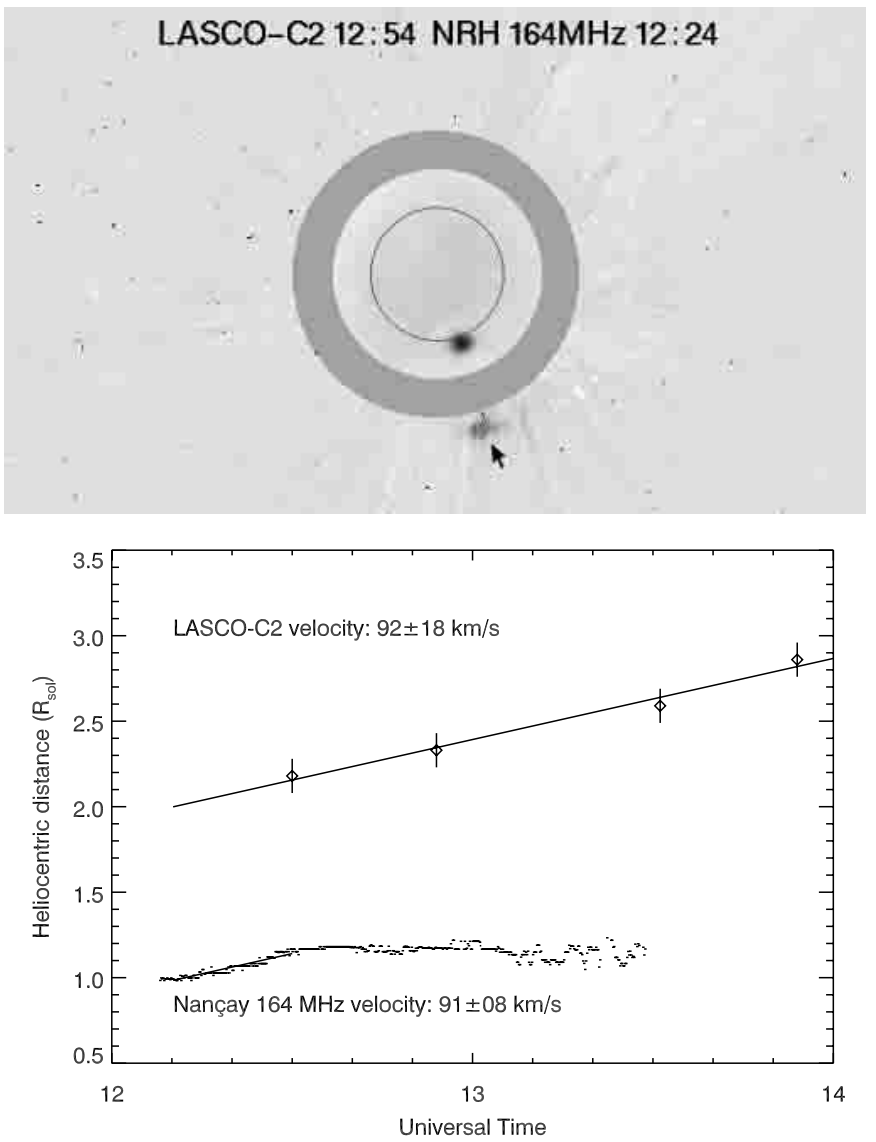

Fig. 8. Upper panel: emerging structure seen on LASCO-C2 (running difference images) and with the NRH. Lower panel: velocity comparison of LASCO-C2 and Nançay observations. The radio velocity was computed between 12:07 and 12:30 UT during the outward motion of the noise storm.

an accuracy of $\sim 0.05 R_{\odot}$ reveal that the decimetric components lie near the feet of these arcades, south to the neutral line delineated by filament a. On account of the projection effects, the metric component seems to be located among medium scale loops southward of AR 8679. These loops gradually disappear during the eruption, as revealed by the difference image presented in Fig. 9. The dark area south of AR 8679 probably indicates an expansion of the loops, which is compatible with the EIT movie of the event and the dimming observed in this region.

Large scale magnetic field lines are involved in the course of the event, for example the transequatorial loops. They are activated before the eruption and during the event, and their southern extremity is very close to the previous arcades. These kinds of loops are thought to be the result of reconnective processes occurring in the corona (Tsuneta 1996; Fárník et al. 1999). They are also commonly associated with the emergence of magnetic flux, such as AR 8686 born on August 28th, and growing in size and complexity up to the eruption. These loops appear on September 1st on YOHKOH images linking the

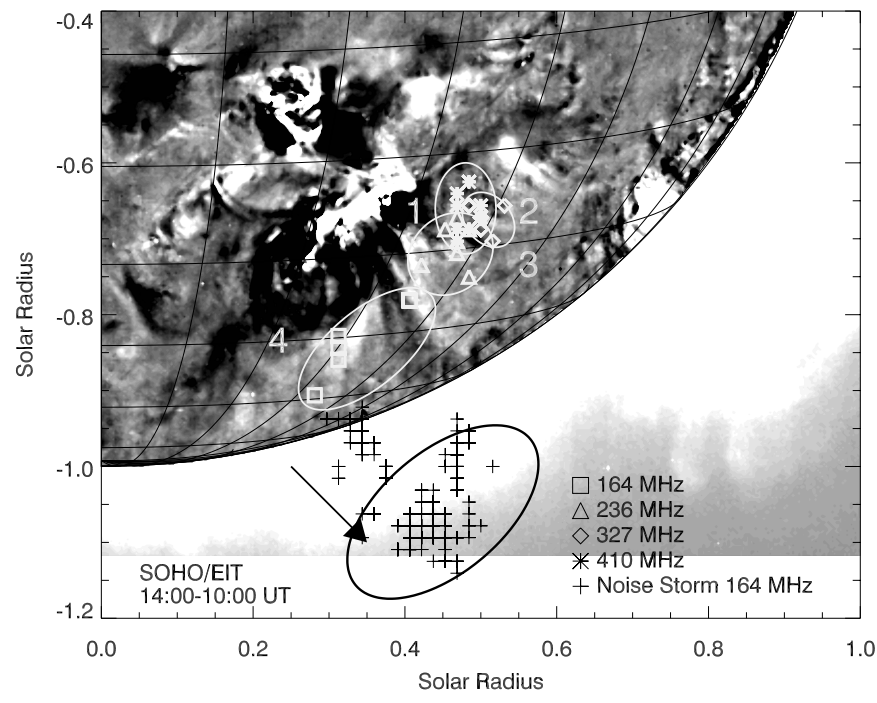

Fig. 9. Difference image of EIT at $195 \AA$ between 14:00 and 10:00 UT, taking into account the solar rotation, together with radio observations performed prior and during the eruption. Numbered ellipses mark the bounds of burst A locations with time at respectively 410, 327, 236 and $164 \mathrm{MHz}$. Ascending motion of the meter noise storm ( + signs) is indicated by the thick arrow. Portion of the image out of the disk is over exposed and not rotated.

neighborhood of AR 8686 and 8677, and are visible the next day on EIT images $(195 \AA)$.

The event under study has different manifestations spread over a large area, comprising the filament c, AR 8686, and AR 8679. Over two days, the magnetic flux increases in AR 8686, "pushing" the filament e westward. A few hours before the dynamical DB, activity of filament $\mathbf{c}$ is closely related to the activation of filament $\mathbf{e}$, and it partially disappears during the eruption. A possible scenario may be the following: as the magnetic flux grows in AR 8686 it pushes out the overlying magnetic structures. A few hours before the eruption, these structures reach an unstable state as revealed by the kinking of transequatorial loops. As the swelling of the entire system goes on (indicated by the slow rising of filament $\mathbf{e}$ ), some of the arcades reconnect, leading to the radio emission and the eruption of filament e. This can explain the faint flare loops linking AR 8679 and AR 8686 and the vicinity of filament a.

\subsection{Dimmings}

Transient Coronal Holes ( $\mathrm{TCH})$ that appear after the event are thought to be a good indication of large scale loops disturbed or destroyed by DBs. Many observations of coronal dimmings associated with X-ray flares and CME onsets have been reported (Rust \& Hildner 1976). Among them, some are relative to transient coronal holes in the vicinity of S-shaped erupting loops (Manoharan et al. 1996; Sterling \& Hudson 1997; Zarro et al.1999). No Sshaped loop is observed in our case prior the event and the 
spatial scales are different. Nevertheless, the post-event loop system described after the eruption of filament $\mathbf{e}$ is apparently not potential, and the activity in regions 8686 and 8679 persists (south part of filament $\mathbf{b}$ of AR 8679 erupts after 15:00 UT). TCH are thought to be the remnants of large scale erupting loops, whose feet were anchored in their neighborhood. Manoharan et al. (1996) explicitly suggest these holes could be created by reconnective processes involving expanding loops from flare location and large scale loops overlying the region (see also Delannée \& Aulanier 2000).

A possible counterpart of the EUV dimming may be found in the radio depression that decimetric observations show to expand after the eruption. As explained before, the filament under study is seen as a depression before the event. The nature of this depression is not fully understood at present, and it is beyond the scope of this paper to determine it. The post eruptive depression presents some differences to the filament-associated one. Its total length, as illustrated in Fig. 6, is three times that of the pre eruptive $\operatorname{dip}\left(0.7 R_{\odot}\right.$ against $\left.0.2 R_{\odot}\right)$, while its depth is $\sim 2 \times 10^{5} \mathrm{~K}$ lower at $327 \mathrm{MHz}$. With a very simple hypothesis, it is possible to estimate the mass loss required to explain the radio dimming. The brightness temperature is given by

$T_{\mathrm{b}}=T_{\mathrm{c}}\left(1-\mathrm{e}^{-\tau}\right)$

and

$\tau=\int_{0}^{\infty} \zeta \frac{n_{\mathrm{e}}^{2}}{T_{\mathrm{c}}^{3 / 2} f^{2}} \mathrm{~d} z$

with $\zeta=0.2$ (Kundu 1965). Assuming $T_{\mathrm{c}}=10^{6} \mathrm{~K}, n_{\mathrm{e}}=$ $n_{0} \exp \left(-z / z_{\mathrm{h}}\right), z_{\mathrm{h}} \sim 0.07 R_{\odot}$, we have:

$\tau=A(f) n_{0}^{2}$ and $\Delta n_{0} \sim \frac{\Delta T_{\mathrm{b}}}{2 A(f) n_{0} T_{\mathrm{c}} \mathrm{e}^{-\tau}}$

where $\Delta n_{0}$ is the change of the electronic density within the dimming area.

With $A(327 \mathrm{MHz}) \sim 4.7 \times 10^{-18} \mathrm{~cm}^{6}$ and $\Delta T_{\mathrm{b}} \sim$ $2 \times 10^{5} \mathrm{~K}$, we get $\Delta n_{0} \sim 1.2 \times 10^{8} \mathrm{~cm}^{-3}$ (for $\tau=1$ ). The area covered by the dip is $S \sim 0.7 \times 0.1 R_{\odot}^{2}$. This gives a mass of $\Delta m \simeq S \times \Delta n_{0} \times \int_{0}^{\infty} \exp \left(-z / z_{\mathrm{h}}\right) \mathrm{d} z \simeq 3 \times 10^{14} \mathrm{~g}$.

This estimation is smaller than the masses of some $10^{15} \mathrm{~g}$ found from CME observations by the SOLWIND (Howard et al. 1985) or SMM (Hundhausen et al. 1994) coronagraphs. It is at the lower limit of CME masses observed by LASCO (Vourlidas et al. 2000), and no CME appears within the LASCO field of view. Since the time scale of the radio depression expansion is about one hour, we speculate that the low mass flux makes the event undetectable by LASCO. However, a thorough analysis of the LASCO sensitivity would be necessary to confirm this hypothesis.

\subsection{Noise storm}

As already mentioned in a previous section, the behavior of the noise storm is closely related to the appearance of a faint expanding structure within the LASCO field of view. Since the radio emission occurs at the plasma frequency level $\left(n_{\mathrm{e}} \sim 3.3 \times 10^{8} \mathrm{~cm}^{-3}\right.$ at $\left.164 \mathrm{MHz}\right)$, the coordinated rise of the noise storm and the white light feature indicates a gradual enhancement of the electronic density with height. The asymptotic altitude reached by the noise storm may indicate that the inner denser regions stop their ascension, while the upper and tenuous parts, unable to support the plasma emission at $164 \mathrm{MHz}$, still rise, as observed on LASCO. The present event is probably not a true CME, but rather reveals a gradual accumulation of plasma. Thus, it could be similar to a process invoked by Kahler \& Hundhausen (1992) to explain streamer reformations after a CME. Opened/expanding structures, inferred from EIT observations of dimmings in the vicinity of AR 8679 in the earlier stages of the event, are closed in a reconnective process occurring at rather high altitude.

\section{Discussion and conclusion}

This article illustrates an example of a filament eruption associated with medium and large scale morphological modifications in the corona, energy release signaled by the $\mathrm{X}$-ray LDE and non thermal radio emission, and mass input into the corona. Nevertheless, all the manifestations are weak, no $\mathrm{H} \alpha$ flare or CME is reported, the X-ray event is small, without hard X-ray counterparts, the radio emission lies only a few million degrees above the quiet sun level, and finally the filament does not seem to be ejected from the sun.

The pre-event long term evolution of filament $\mathbf{e}$ is rather classical, being closely associated with the magnetic flux emergence in a nearby active region (see Feynman \& Martin 1995 for a statistical study). After a slow ascending phase, observed in optical lines, the acceleration of the filament occurs simultaneously with an energy release process, shown by the LDE and the radio burst. The relative timing of the eruption and the manifestations of energy release is rather close to what is described by breakout class models. Antiochos et al. (1999) study the evolution of low lying coronal structures before they erupt. They predict weak reconnection signatures after an initial evolution, occurring far from the main neutral line. Some of these predictions are compatible with our observations: the eruption takes place within a multi polar magnetic system, involving large and small scale magnetic structures. Reconnection is inferred from faint non thermal radio signatures. The location of the "flare" loops and the bursts is far from the place of the eruptive filament. This suggests that each of these manifestations, optical eruption, radio bursts and LDE, are different aspects of a unique, more global magnetic restructuring.

As observed for well developed HXR-microwave bursts (Neupert 1968; Dennis \& Zarro 1993), the particle acceleration, revealed by the non thermal radio emission, occurs during the rising phase of the weak LDE. On a smaller 
energy scale, this is rather similar to other examples linking slow LDEs and non thermal energy releases observed by Hudson \& McKenzie (2000). The weakness of the radio burst suggests that the non thermal electron energy is too low to explain the LDE, but the time coincidence indicates a possible common source.

Large scale magnetic structures are involved in this event. A good example is the transequatorial loops disturbed before and during the event. Contrary to recent observations involving transequatorial loops in CMEs (Khan \& Hudson 2000; Delannée \& Aulanier 2000), they do not seem to play an active role in the eruptive process, but they imply a multipolar magnetic field in the event. Post event manifestations on a large scale are essentially the dimmings seen at EUV and radio wavelengths. As already mentioned, these dimmings are generally associated with mass outflow in the upper corona, for example CMEs. Despite all these favorable indications of a CME, it seems that the event is energetically too small to produce such a coronal disturbance. The mass estimated by radio observations is $\sim 10 \%$ the mass of a typical CME, and no white light counterpart was observed in the LASCO field of view. The progressive disappearance of the radio dimming, beginning at the end of the noise storm, may be attributed to the closing of magnetic field lines opened during the event; this probably indicates the end of the general magnetic restructuring of the area.

Finally, this article illustrates some of the well known links between noise storm onsets and magnetic reconfigurations. In the past, short lived noise storms were found to be associated with filament eruptions (McLean 1973; Lantos et al. 1981; Kundu \& Gopalswamy 1990). In the present case, the location of the noise storm far from the eruptive filament suggests that a global magnetic reconfiguration triggered the noise storm onset. The association between X-ray emissions and noise storms was noticed by Raulin \& Klein (1994) for a sample of nine noise storm onsets and soft X-ray enhancements, other examples being more closely related in duration and temporal evolution (Lantos et al. 1981).

White light coronal enhancements and noise storm onsets were previously described by Kerdraon et al. (1983). They performed a study of several noise storm enhancements related to coronagraphic brightenings seen on $\mathrm{C} / \mathrm{P}$ coronagraph on board SMM spacecraft. They did not mention any motions of the noise storms while velocities of optical features were rather slow $\left(\sim 70 \mathrm{~km} \mathrm{~s}^{-1}\right)$ or negligible. In the present case, the noise storm and the LASCO feature have a similar expanding velocity $\sim 80-90 \mathrm{~km} \mathrm{~s}^{-1}$. In the absence of a low altitude coronagraph (the lower limit of the occulting disk is $\sim 2.5 R_{\odot}$ for LASCO and $\sim 1.5 R_{\odot}$ for the $\mathrm{C} / \mathrm{P}$ coronagraph of $\mathrm{SMM}$ ), it is difficult to compare the shape and the structures of the white light features; nevertheless, the present event is very similar to their observations. Due to a low temporal resolution, they could not distinguish between local restructuring in the coronagraph field of view, or a material transport from below. In the present event, the material comes from the lower corona, as inferred by the motion of the LASCO feature and the noise storm, possibly from the underlying AR, which seems to play a crucial role in the noise storm onset.

Acknowledgements. The authors wish to thank J. P. Delaboudinière for access to EIT data, as well as helpful comments on this manuscript. They would like also to thank A. Raoult for discussions about a preliminary version of this article. The LASCO data were provided by the Naval Research Laboratory (USA). LASCO was constructed by a consortium of the Naval Research Laboratory (USA), Max-Planck-Institut für Aeronomie (Germany), Laboratoire d'Astronomie Spatiale (France), and the University of Birmingham (UK). soHo is a project of international cooperation between ESA and NASA. GOES data were provided by the Solar Data Analysis Center at NASA/GSFC. YОнкоH data used in this work were provided by the Yohkoh Data Archive Centre, at MSSL. The Nançay Radioheliograph is funded by the French Ministry of Education, the CNRS and the Région Centre.

\section{References}

Acton, L., Bruner, M., Brown, W., Lemen, J., \& Hirayama, T. 1988, Adv. Space Res., 8, 93

Alissandrakis, C. E., \& Lantos, P. 1996, Sol. Phys., 165, 61

Antiochos, S., Devore, C., \& Klimchuk, J. 1999, ApJ, 510, 485

Brueckner, G. E., Howard, R. A., Koomen, M. J., et al. 1995, Sol. Phys., 162, 357

d'Azambuja, M., \& d'Azambuja, L. 1948, in Ann. Obs. ParisMeudon, vol. VII, 6

Delaboudinière, J. P., Artzner, G. E., Brunaud, J., et al. 1995, Sol. Phys., 162, 291

Delannée, C., \& Aulanier, G. 2000, Sol. Phys., 190, 107

Delouis, J. M. 1999, Ph.D. Thesis, Université Paris VII

Dennis, B. R., \& Zarro, D. M. 1993, Sol. Phys., 146, 177

Elgarøy, O. 1977, Solar noise storms 1st ed. (Oxford; New York: Pergamon Press), 43

Fárník, F., Karlický, M., \& Švestka, Z. 1999, Sol. Phys., 187, 33

Feynman, J., \& Martin, S. F. 1995, J. Geophys. Res., 100, 3355

Hiei, E., Ishiguro, M., Kosugi, T., \& K., S. 1986, in Coronal and Prominence Plasma, NASA Conf. Publ., 2442, 109

Howard, R. A., Sheeley, N. R., Michels, D. J., \& Koomen, M. J. 1985, J. Geophys. Res., 90, 8173

Hudson, H. S., \& McKenzie, D. E. 2000, in High Energy Solar Physics: Anticipating HESSI, ed. R. Ramaty, \& N. Mandzhavidze, ASP Conf. Ser., 206, 221

Hudson, H. S., \& Ohki, K. 1972, Sol. Phys., 23, 155

Hundhausen, A. J. 1999, in The Many faces if the Sun, ed. K. Strong, J. Saba, B. Haisch, \& J. T. Schmelz (Springer), 143

Hundhausen, A. J., Stanger, A. L., \& Serbicki, S. A. 1994, in Solar Dynamic Phenomena and Solar Wind Consequences, SP-373, ESA, 409

Kahler, S., \& Hundhausen, A. 1992, J. Geophys. Res., 97, 1619 Kerdraon, A., \& Delouis, J. M. 1997, in Coronal Physics from Radio and Space Observations, ed. G. Trottet, 483

Kerdraon, A., Pick, M., Trottet, G., et al. 1983, ApJL, 265, 19 
Khan, J., \& Hudson, H. 2000, Geophys. Res. Lett., 27, 1083 Kundu, M. 1965, Solar Radio Astronomy (Interscience Publishers), 342

Kundu, M. R., \& Gopalswamy, N. 1990, Sol. Phys., 129, 133

Lantos, P., \& Alissandrakis, C. E. 1999, A\&A, 351, 373

Lantos, P., Kerdraon, A., Rapley, G. G., \& Bentley, R. D. 1981, A\&A, 101, 38

Manoharan, P. K., van Driel-Gesztelyi, L., Pick, M., \& Démoulin, P. 1996, ApJL, 468, 73

Marqué, C., Lantos, P., Delouis, J. M., \& Alissandrakis, C. E. 1999, in Proc. 8th SOHO Workshop, ESA SP-446, 483

McLean, D. 1973, Proc. Astron. Soc. Aust., 2, 222

Neupert, W. 1968, ApJL, 153, 59

Pallavicini, R., Serio, S., \& Vaiana, G. S. 1977, ApJ, 216, 108
Raulin, J. P., \& Klein, K.-L. 1994, A\&A, 281, 536

Rust, D., \& Hildner, E. 1976, Sol. Phys., 48, 381

Scherrer, P. H., Bogart, R. S., Bush, R. I., et al. 1995, Sol. Phys., 162, 129

Sterling, A. C., \& Hudson, H. S. 1997, ApJL, 491, L55

Tsuneta, S. 1996, ApJ, 456, L63

Uchida, Y. 1998, in ASP Conf. Ser. 150, IAU Colloq. 167, New Perspectives on Solar Prominences, 163

Vourlidas, A., Subramanian, P., Dere, K., \& Howard, R. 2000, ApJ, 534, 456

Webb, D. 1998, in ASP Conf. Ser. 150, IAU Colloq. 167, New Perspectives on Solar Prominences, 463

Zarro, D. M., Sterling, A. C., Thompson, B. J., Hudson, H. S., \& Nitta, N. 1999, ApJL, 520, 139 